\title{
Registro Electrónico de Adherencia al Tratamiento de Esquizofrenia en Latinoamérica (e-STAR): Resultados clínicos del uso de risperidona inyectable de liberación prolongada a dos años de seguimiento
}

\author{
Rogelio Apiquian, ${ }^{1}$ Rodrigo Córdoba, ${ }^{2}$ Mario Louzã ${ }^{3}$ Ana Fresán ${ }^{4}$
}

Artículo original

\section{SUMMARY}

Schizophrenia is a chronic psychiatric disorder associated to high healthcare costs mainly driven by inpatient care. Lack of adherence to antipsychotic treatment is a common reason for relapse and rehospitalization leading to poor prognosis and global functional impairment of patients. Risperidone long-acting injection (RLAl) has demonstrated its efficacy in treating symptoms of schizophrenia and offers the potential to improve adherence to treatment.

\section{Objective}

To determine clinical and functional efficacy of RLAl and use of health resources (eg., hospitalizations) in a 2-year follow up study among patients with schizophrenia from Latin America.

\section{Method}

The electronic Schizophrenia Treatment Adherence Registry (e-STAR) is an observational study of patients who start treatment with RLAI. Data from patients recruited in Mexico, Colombia and Brazil were collected retrospectively for one year prior to baseline, at baseline and every three months for 24 months. Hospitalization rates and treatment regime were registered. Efficacy was assessed using the Clinical Global Impression of Illness-Severity Scale (CGI-S), while the Global Assessment of Functioning (GAF) and the Personal and Social Performance (PSP) were used for the evaluation of functioning.

\section{Results}

Seventy-three patients completed the two-year follow-up. The proportion of patients hospitalized declined from $16.4 \%$ before treatment to $4.1 \%$ after 2 years of treatment with RLAI. Only $2.7 \%$ discontinued the treatment due to lack of efficacy. Significant improvements were reported in illness severity as well as in global functioning assessed by the CGI-S, GAF and PSP scales, respectively.

\section{Discussion}

Our results give further support of the efficacy of RLAl for the treatment of schizophrenia. Additional to symptom severity reduction and functional recovery, improved treatment adherence and reduced hospitalization rates were observed with the use of RLAI. In a real world clinical setting, RLAl offer an effective long-term treatment for patients with schizophrenia, with a lower use of healthcare resources.

Key words: Risperidone long-acting injection, schizophrenia, Latin America, treatment adherence.

\section{RESUMEN}

La esquizofrenia genera elevados costos al sistema de salud. La falta de adherencia al tratamiento es una de las principales causas de recaídas y hospitalizaciones en la esquizofrenia. Lo anterior conduce a un pobre pronóstico y deterioro funcional de los pacientes. La risperidona inyectable de liberación prolongada (RILP) ha demostrado su eficacia en el tratamiento de la esquizofrenia, ofreciendo la posibilidad de que los pacientes tengan una mayor adherencia terapéutica.

\section{Objetivo}

Determinar la eficacia y efecto sobre la funcionalidad y el uso de recursos hospitalarios de la RILP en una muestra de pacientes con esquizofrenia de América Latina a dos años de seguimiento.

\section{Método}

El Registro Electrónico de Adherencia al Tratamiento de Esquizofrenia en Latinoamérica (e-STAR) es un estudio observacional del uso de la RILP en la esquizofrenia. Se reclutaron pacientes de México, Colombia y Brasil. Se registró la información clínica del paciente un año previo al inicio del tratamiento con la RILP y de forma prospectiva cada tres meses hasta cumplir los 24 meses de seguimiento. Se registraron las hospitalizaciones y el esquema de tratamiento con la RILP. La escala de Impresión Clínica Global-Gravedad (CGI-S) se utilizó como indicador de eficacia mientras que la Escala Global de Funcionamiento (GAF) y la Escala de Desempeño Personal y Social (PSP) se utilizaron para evaluar el funcionamiento.

\section{Resultados}

Setenta y tres pacientes completaron los dos años de seguimiento. La proporción de pacientes hospitalizados disminuyó del 16.4 al $4.1 \%$ después de dos años de tratamiento con la RILP. El $2.7 \%$ descontinuó el tratamiento debido a falta de eficacia. Se observó una mejoría significativa en cuanto a la gravedad del padecimiento y el funcionamiento global.

\section{Discusión}

En la práctica clínica cotidiana, la RILP resulta ser un tratamiento a largo plazo efectivo para la esquizofrenia con el beneficio adicional de una menor utilización de recursos del sistema de salud.

Palabras clave: Risperidona inyectable de liberación prolongada, esquizofrenia, América Latina, adherencia al tratamiento.

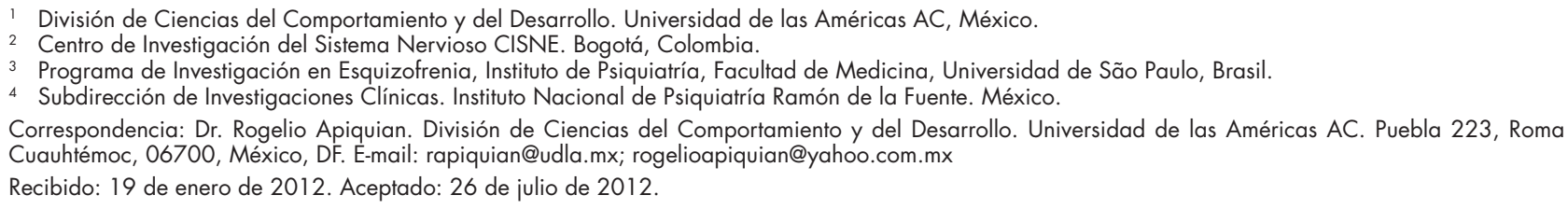




\section{INTRODUCCIÓN}

El tratamiento de los padecimientos mentales no sólo tiene un impacto dentro del sector salud, ya que existen otras áreas afectadas que generan altos costos económicos para la sociedad, tales como la disminución o carencia de productividad y el ausentismo laboral, entre otros. Se ha reportado que la esquizofrenia es uno de los trastornos mentales que genera mayores costos al sistema de salud. ${ }^{1}$ Se estima que el costo anual total de la esquizofrenia para países latinoamericanos y el Caribe es de 1.81 en millones de dólares por millón de personas. ${ }^{2,3}$

Los costos directos en la atención de la esquizofrenia se derivan principalmente de las hospitalizaciones y la atención brindada en instituciones de salud. Se estima que el costo de los medicamentos tan sólo representa entre $1.1 \%$ y $9 \%$ del costo directo de la enfermedad ${ }^{4,5}$ No obstante, el incremento en hospitalizaciones psiquiátricas, así como un mayor uso de los recursos generales del sistema de salud, generalmente son consecuencia de la falta de adherencia que muestran los pacientes al tratamiento a largo plazo. ${ }^{6-8}$

Al menos entre 40 y 50\% de los pacientes con esquizofrenia muestran una pobre adherencia al tratamiento con antipsicóticos. ${ }^{79-11} \mathrm{El}$ olvidar tomar el medicamento, pensar que el medicamento no es necesario, la percepción subjetiva de falta de eficacia o la intolerancia a los efectos secundarios son algunas de las razones por las cuales los pacientes abandonan el tratamiento con antipsicóticos. ${ }^{12-14}$

La falta de adherencia es particularmente elevada cuando el tratamiento es con antipsicóticos orales, ${ }^{7}$ incluso superior a $70 \%$ en los primeros 18 meses de tratamiento, ${ }^{15}$ lo que lleva a replantear el manejo del tratamiento a largo plazo para la esquizofrenia. Algunos estudios reportan que los pacientes con poca adherencia a los medicamentos orales muestran una mejor adherencia a los medicamentos inyectables de liberación prolongada. ${ }^{16,17}$ De forma particular, se destaca el papel de los antipsicóticos atípicos de liberación prolongada, ya que brindan adecuados parámetros de eficacia y seguridad, así como el beneficio de una mayor adherencia debido a su mecanismo de liberación. ${ }^{12,18}$

El primer antipsicótico atípico inyectable de liberación prolongada fue la risperidona (RILP). ${ }^{19}$ La RILP ha mostrado su eficacia y tolerabilidad en diversos ensayos clínicos, ${ }^{20-23}$ observándose además un menor número de hospitalizaciones y una mayor adherencia al tratamiento por parte de los pacientes. ${ }^{24-28}$

La mayoría de los datos publicados en relación con la eficacia de los antipsicóticos proviene de ensayos clínicos controlados. La rigidez metodológica de estos estudios permite obtener evidencia rigurosa en torno al medicamento pero limita la aplicabilidad de estos resultados en la práctica clínica cotidiana. ${ }^{27,29,30}$ Debido a esto, se ha propuesto llevar a cabo estudios observacionales, con adecuados diseños metodológicos, encaminados a valorar la eficacia de tales medicamentos en la práctica clínica cotidiana.
El Registro Electrónico de Adherencia al Tratamiento de Esquizofrenia (e-STAR) ${ }^{31}$ es un registro internacional de tipo observacional del uso de la risperidona inyectable de liberación prolongada (RILP) en la práctica clínica cotidiana en un periodo de seguimiento a 24 meses. Adicionalmente, se registra la información un año previo al inicio de la RILP. El objetivo primario de este estudio es evaluar la eficacia y efecto del tratamiento a largo plazo con la RILP sobre la funcionalidad y el uso de recursos hospitalarios en una muestra de pacientes con esquizofrenia tratados en un contexto clínico cotidiano.

El presente estudio forma parte del e-STAR y presenta los resultados de aquellos pacientes que iniciaron su tratamiento con RILP y se contó con información a los dos años de seguimiento provenientes de tres países de América Latina: México, Colombia y Brasil.

\section{MATERIAL Y MÉTODOS}

El estudio fue aprobado por los Comités de Ética y de Investigación de las instituciones participantes en cada país. A todos los pacientes, incluido al familiar responsable o representante legal, se les informó verbalmente de los procedimientos a realizar y se solicitó su consentimiento por escrito para su inclusión en el estudio, asegurando la confidencialidad y aprobación para reportar los resultados. En el caso de los pacientes que debido a la gravedad de los síntomas de la enfermedad no pudieran tener un adecuado entendimiento de las condiciones del estudio, se solicitó el consentimiento informado del familiar responsable o representante legal.

\section{Pacientes}

Se incluyeron pacientes con el diagnóstico de esquizofrenia y trastorno esquizoafectivo de acuerdo con los criterios diagnósticos del DSM-IV. ${ }^{32}$ Los pacientes fueron reclutados de los servicios de hospitalización, consulta externa y salas de urgencias de instituciones públicas y privadas de México, Colombia y Brasil. Se incluyeron pacientes mayores de 18 años, que presentaran síntomas psicóticos activos y pudieran beneficiarse del uso de RILP de acuerdo con los criterios clínicos del investigador. Se excluyeron los pacientes con antecedente de intolerancia o pobre respuesta clínica a la risperidona no asociada a la falta de adherencia y a mujeres en edad fértil que planearan embarazarse durante el periodo de seguimiento del estudio.

De acuerdo con estos criterios, se contó con un total de 151 pacientes provenientes de México, Colombia y Brasil. Sin embargo, el registro electrónico de los datos basales y de seguimiento de 78 pacientes se encontraba incompleto, sin que esto se reportara asociado a abandonos del estudio o eficacia limitada de la RILP. Para el presente estudio, sólo se incluyeron en el análisis aquellos pacientes que iniciaran 
tratamiento con la RILP y contaran con información de su uso a los 24 meses de seguimiento.

\section{Instrumentos}

- Impresión Clínica Global, Gravedad-CGI-S (Clinical Global Impression-Severity). El CGI-S consiste en un solo reactivo en el que el clínico asigna una calificación global con una escala que va del 0 al 6, donde se califica la gravedad global del padecimiento del paciente. La validez del instrumento está dada por la asumida capacidad clínica del evaluador para determinar el estado del paciente. ${ }^{33}$

- Evaluación Global de Funcionamiento-GAF (Global Assessment of Functioning). El GAF es una de las formas de evaluación global más ampliamente utilizadas en psiquiatría; es una escala continua de 1 a 100 puntos, donde el clínico evalúa en forma general el estado del paciente en las áreas de funcionamiento psicológico, social y ocupacional. El puntaje máximo es indicativo de la ausencia de síntomas y un alto funcionamiento, mientras que el puntaje mínimo implica que existe peligro de dañarse a sí mismo o a otros, y/o una incapacidad persistente para mantener la higiene personal mínima. ${ }^{32,34}$

- Escala de Desempeño Personal y Social - PSP (Personal and Social Performance Scale). La PSP es un instrumento breve de un reactivo, que evalúa del 1 al 100 el funcionamiento del paciente de acuerdo con cuatro indicadores principales: 1. actividades socialmente útiles, 2. relaciones personales y sociales, 3 . autocuidado y 4 . conductas perturbadoras y agresivas. Cada uno de éstos es calificado en una escala Likert de 6 grados de gravedad (ausente-extremadamente grave). Los resultados de esta valoración se transforman en la puntuación global del instrumento. ${ }^{35,36}$

\section{Procedimiento}

Para que el diseño del estudio no interfiriera con los procedimientos llevados a cabo en la práctica clínica cotidiana, el manejo de los pacientes fue llevado a cabo exclusivamente por el médico tratante. Una vez que el médico y el paciente o su representante legal acordaron iniciar el tratamiento de la enfermedad con la RILP, se recababa la historia clínica del paciente así como su progreso con la RILP, la cual era documentada y capturada en el sistema electrónico del e-STAR.

En la fase basal del estudio se recolectaron los datos demográficos, diagnóstico principal, duración de la enfermedad, razones para iniciar el tratamiento con la RILP y la dosis inicial, gravedad global del padecimiento (CGI-S) y nivel de funcionalidad (GAF y PSP). Posteriormente se realizaron evaluaciones trimestrales hasta cumplir los 24 meses de seguimiento. Como indicadores de la eficacia de la RILP se emplearon el CGI-S, el GAF y el PSP en cada evaluación. Adicionalmente, se registró el uso de servicios de hospita- lización psiquiátrica durante el periodo de seguimiento, el cual se comparó con el número de hospitalizaciones reportadas en el periodo retrospectivo del e-STAR.

La dosis de RILP fue ajustada durante el seguimiento y de acuerdo con el criterio clínico del médico tratante. De la misma forma, se registraron tanto el uso de tratamientos concomitantes como la adherencia al tratamiento con RILP, esta última de acuerdo con la apreciación general del médico tratante.

\section{Análisis estadístico}

La descripción de las variables incluidas en el estudio se realizó con frecuencias y porcentajes en el caso de las categóricas y con medias y desviaciones estándar (D.E.) para las continuas. La prueba McNemar fue utilizada para comparar las evaluaciones de seguimiento vs. evaluaciones retrospectivas en relación con las hospitalizaciones y el uso de medicamentos concomitantes. Para determinar la eficacia se realizaron $t$ de Student para muestras pareadas (evaluaciones de seguimiento vs. basal) con las puntuaciones en las escalas del CGI, GAF y PSP. El nivel de significancia estadística se fijó con una $\mathrm{p} \leq 0.05$, con intervalos de confianza de $95 \%$.

\section{RESULTADOS}

a) Características demográficas y clínicas de los pacientes. De los 73 pacientes que recibieron RILP en la fase basal y que contaron con información de su uso, a los 24 meses de seguimiento, 61 fueron reclutados en México, ocho en Colombia y cuatro en Brasil. El 68.5\% fueron hombres, con una edad promedio de 33.2 (D.E.=9.3) años. El 85\% ( $n=62)$ de los pacientes se encontraba sin un empleo económicamente remunerado al inicio del estudio. La mayoría de los pacientes tuvieron el diagnóstico de esquizofrenia $(n=69,93.2 \%)$ y los pacientes restantes $(n=4,5.5 \%)$ fueron diagnosticados con trastorno esquizoafectivo. El tiempo de evolución promedio del padecimiento fue de 9.7 (D.E.=8.3) años.

Las tres principales razones para iniciar el tratamiento con RILP fueron: falta de respuesta al tratamiento previo $(n=27,37 \%)$, falta de adherencia $(n=22,30.1 \%) y$ para ser empleado como tratamiento de mantenimiento $(\mathrm{n}=10,13.7 \%)$.

b) Adherencia al tratamiento con RILP. De los 73 pacientes incluidos, un paciente descontinuó el tratamiento con RILP en el transcurso de los dos años de seguimiento. El tiempo en el que se descontinuó la RILP fue de 368 días. La razón por la que se descontinuó el uso de la RILP fue por falta de respuesta clínica de acuerdo con el criterio del médico tratante.

En la evaluación basal, 82.2\% $(n=60)$ recibieron una dosis de $25 \mathrm{mg}$ de RILP y $17.8 \%(\mathrm{n}=13)$ de $37.5 \mathrm{mg}$; a los dos 
Cuadro 1. Pacientes hospitalizados al menos en una ocasión por período evaluado y estancia intrahospitalaria $(n=73)$

\begin{tabular}{ccccccccc}
\hline & \multicolumn{2}{c}{$\begin{array}{c}\text { Pacientes hospitalizados }(\mathrm{n}=73) \\
\mathrm{n}(\%)\end{array}$} & & \multicolumn{3}{c}{$\begin{array}{c}\text { Estancia hospitalaria total (días) } \\
\text { Media (D.E.) }\end{array}$} \\
\cline { 2 - 4 } \cline { 6 - 7 } Período & \multicolumn{2}{c}{ Retrospectivo } & Prospectivo & Valor $\mathrm{p}^{*}$ & & Retrospectivo & Prospectivo & Valor $\mathrm{p}^{* *}$ \\
\hline 3 meses & $11(15.1)$ & 0.0 & $<0.001$ & & $3.9(11.6)$ & 0.0 & 0.005 \\
6 meses & $15(20.5)$ & $1.0(1.4)$ & $<0.001$ & & $8.2(22.9)$ & $0.7(6.3)$ & 0.006 \\
12 meses & $20(27.4)$ & $3.0(4.1)$ & $<0.001$ & & $16.2(48.9)$ & $1.1(6.7)$ & 0.010 \\
18 meses & NA & $4.0(5.5)$ & - & & NA & $1.5(7.4)$ & - \\
24 meses & NA & $5.0(6.8)$ & - & & NA & $1.5(7.4)$ & - \\
\hline
\end{tabular}

NA Evaluaciones retrospectivas no incluidas en la metodología del estudio.

* Valores obtenidos con la prueba de McNemar.

* * Valores obtenidos con la prueba de + de Student para muestras pareadas.

años de seguimiento, 35 pacientes $(48.6 \%)$ se encontraban con $25 \mathrm{mg}$, 18 (25\%) con $37.5 \mathrm{mg}$ y 19 (26.4\%) con $50 \mathrm{mg}$.

La adherencia al tratamiento con RILP en ambas evaluaciones fue reportada como excelente (de 81 al 100\% del cumplimiento) de acuerdo con la apreciación general del médico tratante en todos los pacientes. Además, a los dos años de seguimiento, 96.4\% de los pacientes reportó encontrarse muy satisfecho con el tratamiento con RILP; en cambio, en la fase basal del estudio sólo $35.7 \%$ de éstos se encontraba satisfecho con el tratamiento anterior a la RILP.

c) Uso de recursos - Hospitalización y medicamentos concomitantes. En contraste con el periodo retrospectivo de evaluación (12 meses), se observó una reducción significativa en el número de pacientes que fueron hospitalizados, así como el tiempo promedio total de estancia intrahospitalaria una vez que los pacientes fueron tratados con RILP. La proporción de pacientes disminuyó de 16.4 a $4.1 \%$ ( $p<0.02)$, y el tiempo de estancia intrahospitalaria total mostró una reducción promedio de 11.2 días $(p=0.03)$. Estos resultados se muestran en el cuadro 1.

En cuanto al uso de medicamentos concomitantes, se contó con el registro de la información de 56 pacientes tanto en la evaluación basal como a los dos años de seguimiento. Se observó un mayor número de pacientes que se encontraban sin medicamentos adicionales al final del seguimiento. De forma específica hubo una reducción en el uso de anticolinérgicos (cuadro 2).

d) Gravedad sintomática y funcionalidad. En la etapa basal del estudio, $77.7 \%$ de los pacientes presentaron síntomas moderados/marcados (CGI-S de 4-6 puntos), porcentaje que se redujo a $23.8 \%$ a los dos años de seguimiento. Se observó una mejoría global en la gravedad de los síntomas a lo largo del estudio, mejoría que se presentó desde el tercer mes de tratamiento con la RILP, manteniéndose de forma estable hasta el mes 24 (gráfica 1).

Desde el tercer mes de seguimiento se reportó una mejoría en la funcionalidad de los pacientes. Lo anterior quedó evidenciado por la obtención de mayores puntajes en las escalas GAF y PSP (gráfica 2) desde el tercer mes de seguimiento; la recuperación funcional se mantuvo estable a lo largo éste.

Un indicador adicional de la mejoría en la funcionalidad fue la situación laboral; se contó con información de 56 pacientes tanto en la evaluación basal como a los dos años de seguimiento. Al inicio del estudio, sólo 10 pacientes $(17.8 \%)$ tenían una ocupación económicamente remunerada, mientras que a los dos años de seguimiento este porcentaje se incrementó a $39.3 \%(n=22)$.

\section{DISCUSIÓN}

El objetivo del presente estudio fue reportar la eficacia y el

Cuadro 2. Tratamiento concomitante

\begin{tabular}{|c|c|c|c|c|c|c|}
\hline & $\begin{array}{l}\text { Basal } \\
(n=56)\end{array}$ & $\begin{array}{l}\text { Mes } 6 \\
(n=44)\end{array}$ & $\begin{array}{c}\text { Mes } 12 \\
(n=45)\end{array}$ & $\begin{array}{c}\text { Mes } 18 \\
(n=44)\end{array}$ & $\begin{array}{c}\text { Mes } 24 \\
(n=56)\end{array}$ & \\
\hline & $\begin{array}{ll}\mathrm{n} & (\%) \\
\end{array}$ & $n \quad(\%)$ & $\begin{array}{ll}\mathrm{n} & (\%)\end{array}$ & $\begin{array}{ll}\mathrm{n} & (\%) \\
\end{array}$ & $\begin{array}{ll}n & (\%)\end{array}$ & Valor $p^{*}$ \\
\hline Sin medicamentos & $16(28.6)$ & $19(43.2)$ & $21(46.7)$ & $21(47.7)$ & $29(51.8)$ & 0.02 \\
\hline Anticolinérgico & $18(32.1)$ & $10(22.7)$ & $8(17.8)$ & $7(15.9)$ & $6(10.7)$ & 0.01 \\
\hline Antidepresivos & 4 (7.1) & $4 \quad(9.1)$ & $3(6.7)$ & $2(4.5)$ & $4(7.1)$ & 1.00 \\
\hline Estabilizadores del ánimo & $13(23.2)$ & $7(15.9)$ & $8(17.8)$ & $10(22.7)$ & $13(23.2)$ & 1.00 \\
\hline Benzodiazepinas & $20(35.7)$ & $7(15.9)$ & $11(24.4)$ & $11(25.0)$ & $12(21.4)$ & 0.09 \\
\hline Medicamentos somáticos & $6(10.7)$ & $6(13.6)$ & $7(15.6)$ & $7(15.9)$ & $7(12.5)$ & 1.00 \\
\hline
\end{tabular}

Nota' Sólo se incluyeron los pacientes que contaban con la evaluación basal y la de dos años de seguimiento.

Nota $^{2}$ Cada paciente pudo encontrarse con más de un medicamento concomitante en los períodos evaluados.

* Valores obtenidos con la prueba de McNemar entre el mes 24 vs. basal. 


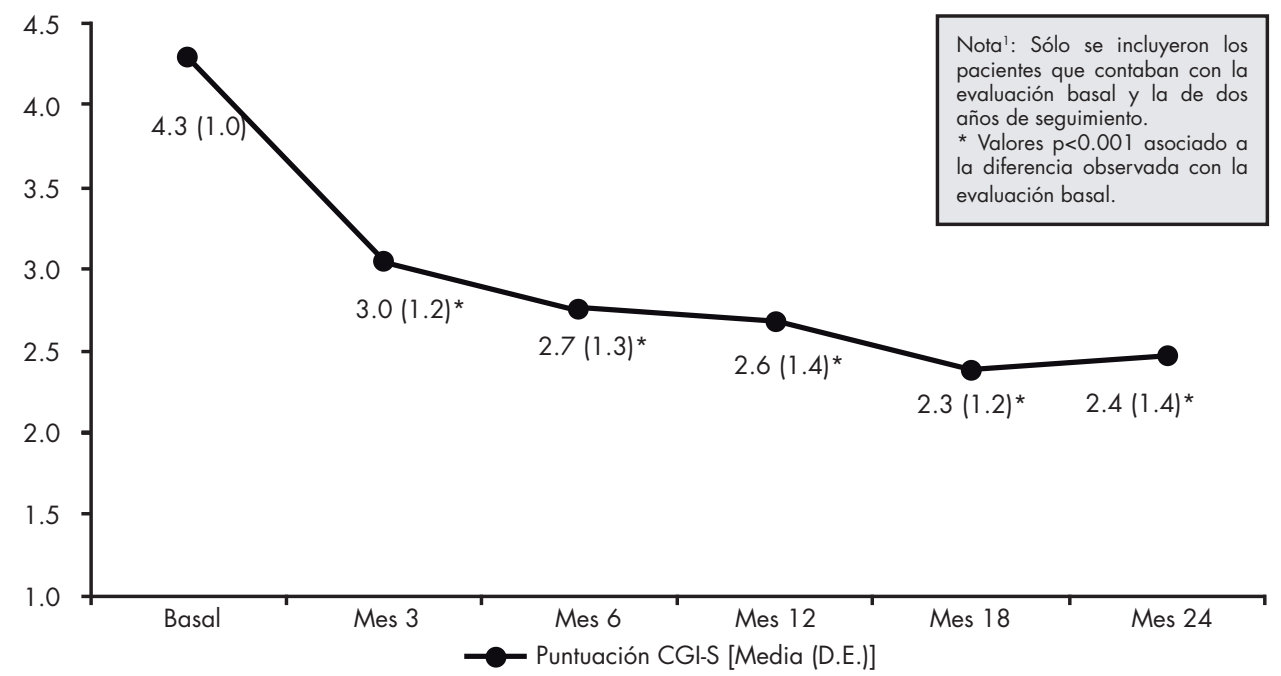

Gráfica 1. Evolución de la gravedad sintomática a lo largo del estudio (CGI-S).

efecto sobre la funcionalidad y el uso de recursos hospitalarios de la RILP a dos años de seguimiento en una muestra de pacientes de América Latina con esquizofrenia.

El estudio apoya y sustenta los resultados descritos en el informe preliminar del e-STAR en América Latina, ${ }^{37}$ en los cuales la RILP muestra una adecuada eficacia sobre el control de los síntomas de la esquizofrenia y la funcionalidad global del paciente. Al inicio del tratamiento con RILP, los pacientes presentaban una gravedad moderada/ marcada de los síntomas psicóticos y dificultades en el funcionamiento psicosocial global. Una vez iniciado el tratamiento con RILP, se observó una clara mejoría sintomática y funcional desde el tercer mes de tratamiento, mejoría que se mantuvo a lo largo de los dos años de seguimiento. Aun cuando la eficacia de la RILP en el manejo de los síntomas de la esquizofrenia ha sido previamente demostrada en ensayos clínicos controlados, los reportes de su eficacia en un estudio observacional realizado en la práctica clínica cotidiana brindan un mayor sustento para su empleo en el tratamiento de mantenimiento requerido por los pacientes con esquizofrenia.

La falta de eficacia con tratamientos previos fue una de las principales razones para la prescripción de la RILP a los pacientes del presente estudio. En América Latina, el uso de antipsicóticos inyectables debe limitarse para aquellos pacientes con síntomas muy graves del padecimiento o con pobre adherencia al tratamiento. ${ }^{37}$ Parece ser que la prescripción de la RILP para los pacientes graves se asocia con una percepción de mayor eficacia terapéutica; sin embargo, esta misma percepción puede llevar a concepciones erróneas sobre los criterios específicos para la prescripción de RILP, antipsicótico que puede ser empleado incluso en pacientes que no han sido previamente tratados con antipsicóticos..$^{37-40}$

Además, los presentes resultados muestran que la RILP

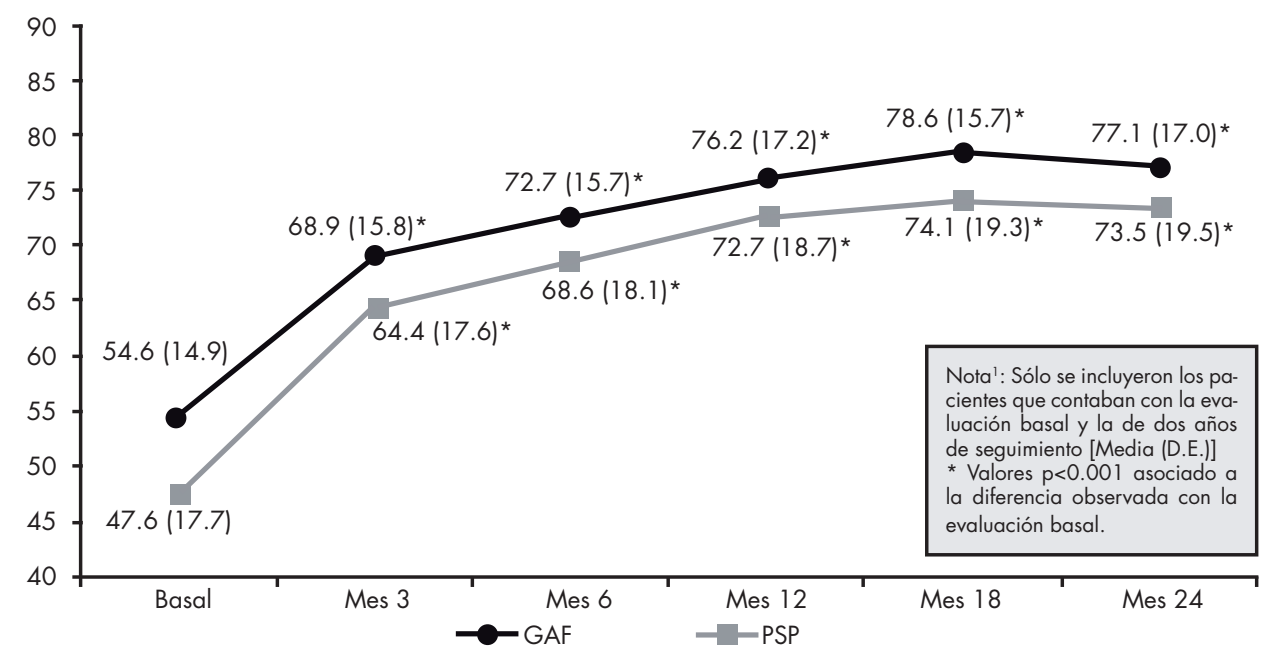

Gráfica 2. Evolución de la funcionalidad a lo largo del estudio. 
es un medicamento eficaz para el tratamiento a largo plazo. En el estudio CATIE (The Clinical Antipsychotic Trials of Effectiveness) se reportó que $74 \%$ de los pacientes descontinúan el uso de antipsicóticos durante los primeros 18 meses de tratamiento, ${ }^{41,42}$ mientras que en este estudio se encontró una baja proporción de pacientes que descontinuaron el tratamiento debido a la falta de eficacia.

La segunda causa para iniciar el tratamiento con RILP en el presente estudio fue la falta de adherencia al tratamiento previo. La falta de adherencia al tratamiento farmacológico en la esquizofrenia se asocia a un mayor deterioro global, secundario a un mayor número de recaídas, exacerbación de síntomas psicóticos y mayor número de hospitalizaciones psiquiátricas a lo largo del padecimiento. ${ }^{12,26,43-46}$ La falta de adherencia al tratamiento no sólo impacta en el manejo de la enfermedad; un mayor número de hospitalizaciones implica mayores costos para el paciente y sus familiares en la atención especializada, así como un mayor uso de recursos del sistema de salud en términos del empleo de instalaciones hospitalarias y de más medicamentos concomitantes. ${ }^{47-50}$

En el manejo de la esquizofrenia no sólo es importante considerar la eficacia de un medicamento en el control de los síntomas psicóticos. Aun cuando los antipsicóticos atípicos representaron un gran avance en al tratamiento de la esquizofrenia, la necesidad de contar con nuevos medicamentos dirigidos a incrementar la adherencia, prevenir recaídas y disminuir el uso de medicamentos concomitantes y uso de servicios del sistema de salud ${ }^{5}$ se convirtió en un reto para los especialistas de la salud mental. Así, con la introducción de los antipsicóticos atípicos inyectables de liberación prolongada, se abrió la posibilidad de contar con un tratamiento eficaz que redujera los costos directos de la enfermedad asociados al uso de recursos hospitalarios. Los resultados que obtuvimos con el uso de la RILP en pacientes de América Latina son consistentes con lo previamente reportado por otros países. La RILP es un tratamiento que, además de ser eficaz en el manejo de los síntomas de la enfermedad, permite una adecuada adherencia al tratamiento por parte de los pacientes, lo que disminuye el número de hospitalizaciones y tiempo de estancia intrahospitalaria. 5, 19,23,27,31,37,51-53

Una de las principales implicaciones de estos resultados se encuentra dirigida a las concepciones en torno al uso de antipsicóticos inyectables como tratamiento de primera elección para la esquizofrenia, incluso en situaciones clínicas no relacionadas con dificultades en la adherencia al tratamiento. Con el uso de la RILP, los pacientes se verían beneficiados por un antipsicótico eficaz para el tratamiento de los síntomas, el cual no tiene que ser tomado diariamente, lo que aumenta las probabilidades de una adecuada adherencia y reduce las posibilidades de una recaída y eventual hospitalización. De esta forma, el funcionamiento global de los pacientes podría mantenerse en un nivel adecuado, permitiéndoles una mejor integración social y laboral en su entorno.
El presente estudio tiene diversos sesgos y limitaciones. Además de los posibles sesgos de selección y de observación propios de un estudio abierto, la principal limitación del estudio se centra en el sesgo de recolección de los datos basales y de seguimiento. Un $48.3 \%$ de los pacientes inicialmente incluidos en el estudio no pudieron ser analizados debido a este sesgo, lo cual limita de forma importante la generalización de los datos obtenidos. Este punto deberá ser vigilado y controlado en otros estudios que se realicen en la práctica clínica cotidiana, inicialmente mediante el fortalecimiento de la interacción entre la atención médica y la investigación, y una vigilancia más estrecha del registro de datos vía electrónica.

Sin embargo, nuestros resultados proporcionan información relevante para el manejo de la esquizofrenia en la práctica clínica cotidiana, con datos adecuados sobre la eficacia y beneficios asociados al uso de recursos con el tratamiento basado en la RILP, replicando lo encontrado por otros estudios internacionales. Por otro lado, aun cuando la cuantificación del beneficio económico no forma parte del objetivo del estudio, nuestros resultados brindan una base para el desarrollo de estudios encaminados a valorar el costo-beneficio de la RILP para el paciente y los sistemas de salud de América Latina.

\section{AGRADECIMIENTO}

Los autores reconocen a los siguientes investigadores principales de los sitios que ingresaron pacientes: Brasil: Helio Helkis, Rodrigo Bressan, Hamilton Grabowski; Colombia: Rodrigo de la Espriella; México: Magdaleno Pérez, Agustín Bañuelos, Carlos Peralta, Patricia Martínez, Lauro Castanedo, Raul Escamilla y Víctor Joffre.

\section{REFERENCIAS}

1. Organization for Economic Co-operation Development. París: Policy Brief. 2008.

2. Cabello H, Díaz Castro L, Arredondo A. Costo-efectividad de intervenciones para esquizofrenia en México. Salud Mental 2011;34:95-102.

3. World Health Organization. Disease control priorities related to mental, neurological, developmental, and substance abuse disorders. Ginebra; 2006.

4. Knapp M, Mangalore R, Simon J. The global costs of schizophrenia. Schizophr Bull 2004;30:279-293.

5. Olivares J, Peuskens J, Pecenak J et al. Clinical and resource-use outcomes of risperidone long-acting injection in recent and long-term diagnosed schizophrenia patients: results from a multinational electronic registry. Curr Med Res Opin 2009;25:2197-2206.

6. Svarstad B, Shireman T, Sweeney J. Using drug claims data to assess the relationship of medication adherence with hospitalization and costs. Psychiatr Serv 2001;52:805-811.

7. Valenstein M, Blow F, Copeland L et al. Poor antipsychotic adherence among patients with schizophrenia: medication and patient factors. Schizophr Bull 2004;30:255-264.

8. Lacro J, Dunn L, Dolder C, Leckband S et al. Prevalence of and risk factors for medication nonadherence in patients with schizophrenia: a comprehensive review of recent literature. J Clin Psychiatry 2002;63:892-909. 
9. Fenton W, Blyler C, Heinssen R. Determinants of medication compliance in schizophrenia: empirical and clinical findings. Schizophr Bull 1997;23:637-651.

10. Velligan D, Lam F, Ereshefsky L, Miller A. Psychopharmacology: Perspectives on medication adherence and atypical antipsychotic medications. Psychiatr Serv 2003;54:665-667.

11. Boaz TL, Constantine RJ, Robst J, Becker MA et al. Risperidone long-acting therapy prescribing patterns and their impact on early discontinuation of treatment in a large Medicaid population. J Clin Psychiatry 2011; 72(8): 1079-1085.

12. Lang K, Meyers J, Korn J et al. Medication adherence and hospitalization among patients with schizophrenia treated with antipsychotics. Psychiatr Serv 2010;61:1239-1247.

13. Liu-Seifert HAD, Kinon BJ. Discontinuation of treatment of schizophrenic patients is driven by poor symptom response: a pooled post-hoc analysis of four atypical antipsychotic drugs. BMC Med 2005;23(3):21.

14. Perkins D. Predictors of noncompliance in patients with schizophrenia. J Clin Psychiatry 2002;63:1121-1128.

15. Lieberman J, Stroup T. The NIMH-CATIE Schizophrenia Study: what did we learn? Am J Psychiatry 2011;168:770-775.

16. Olfson M, Marcus S, Ascher-Svanum H. Treatment of schizophrenia with long-acting fluphenazine, haloperidol, or risperidone. Schizophr Bull 2007;33:1379-1387.

17. West J, Marcus S, Wilk J, Countis L et al. Use of depot antipsychotic medications for medication nonadherence in schizophrenia. Schizophr Bull 2008;34:995-1001.

18. Keith S, Pani L, Nick B et al. Practical application of pharmacotherapy with long-acting risperidone for patients with schizophrenia. Psychiatr Serv 2004;55:997-1005.

19. Chang C, Tzeng D, Lung F. Treatment effectiveness and adherence in patients with schizophrenia treated with risperidone long-acting injection. Psychiatry Res 2010;180:16-19.

20. Chue P, Eerdekens M, Augustyns I et al. Comparative efficacy and safety of long-acting risperidone and risperidone oral tablets. Eur Neuropsychopharmacol 2005;15:111-117.

21. Fleischhacker W, Eerdekens $M$, Karcher $K$ et al. Treatment of schizophrenia with long-acting injectable risperidone: a 12-month open-label trial of the first long-acting second-generation antipsychotic. J Clin Psychiatry 2003;64:1250-1257.

22. Kane J, Eerdekens M, Lindenmayer J, Keith S. Long-acting injectable risperidone: efficacy and safety of the first long-acting atypical antipsychotic. Am J Psychiatry 2003;160:1125-1132.

23. Schmauss M, Sacchetti E, Kahn J, Medori R. Efficacy and safety of risperidone long-acting injectable in stable psychotic patients previously treated with oral risperidone. Int Clin Psychopharmacol 2007;22:85-92.

24. Taylor M, Currie A, Lloyd K, Price M et al. Impact of risperidone long acting injection on resource utilization in psychiatric secondary care. J Psychopharmacol 2008;22:128-131.

25. Lindenmayer J, Parak M, Gorman J. Improved long-term outcome with long-acting risperidone treatment of chronic schizophrenia with prior partial response. J Psychiatr Pract 2006;12:55-57.

26. Llorca P. Partial compliance in schizophrenia and the impact on patient outcomes. Psychiatry Res 2008;161:235-247.

27. Olivares J, Rodriguez-Morales A, Diels J et al. Long-term outcomes in patients with schizophrenia treated with risperidone long-acting injection or oral antipsychotics in Spain: results from the electronic Schizophrenia Treatment Adherence Registry (e-STAR). Eur Psychiatry 2009;24:287-296.

28. Willis M, Svensson M, Löthgren M, Eriksson B et al. The impact on schizophrenia-related hospital utilization and costs of switching to long-acting risperidone injections in Sweden. Eur J Health Econ 2010;11:585-594.

29. Hofer A, Hummer M, Huber R, Kurz M et al. Selection bias in clinical trials with antipsychotics. J Clin Psychopharmacol 2000;20:699-702.
30. Thornley B, Adams C. Content and quality of 2000 controlled trials in schizophrenia over 50 years. BMJ 1998;317:1181-1184.

31. Peuskens J, Olivares J, Pecenak J et al. Treatment retention with risperidone long-acting injection: 24-month results from the Electronic Schizophrenia Treatment Adherence Registry (e-STAR) in six countries. Curr Med Res Opin 2010;26:501-509.

32. American Psychiatric Association. Diagnostic and Statistical Manual of Mental Disorders. Cuarta edición. Washington, DC: 1994.

33. Bech P, Malt U, Dencker S et al. Scales for assessment of diagnosis and severity of mental disorders. Acta Psychiatr Scand 1993;87(supl 372):16-64.

34. Endicott J, Spitzer R, Fleiss J, Cohen J. The global assessment scale. A procedure for measuring overall severity of psychiatric disturbance. Arch Gen Psychiatry 1976;33:766-771.

35. Morosini P, Magliano L, Brambilla L, Ugolini S et al. Development, reliability and acceptability of a new version of the DSM-IV Social and Occupational Functioning Assessment Scale (SOFAS) to assess routine social functioning. Acta Psychiatr Scand 2000;101:323-329.

36. Apiquian R, Ulloa R, Herrera-Estrella $M$ et al. Validity of the Spanish version of the Personal and Social Performance scale in schizophrenia. Schizophr Res 2009;112:181-186.

37. Apiquian R, Córdoba R, Louzã M. Clinical outcomes of long-acting injectable risperidone in patients with schizophrenia: six-month follow-up from the Electronic Schizophrenia Treatment Adherence Registry in Latin America. Neuropsychiatr Dis Treat 2010;7:19-26.

38. Emsley R, Oosthuizen P, Koen L, Niehaus D et al. Remission in patients with first-episode schizophrenia receiving assured antipsychotic medication: a study with risperidone long-acting injection. Int Clin Psychopharmacol 2008;23:325-331.

39. Emsley R, Medori R, Koen L, Oosthuizen P et al. Long-acting injectable risperidone in the treatment of subjects with recent-onset psychosis: a preliminary study. J Clin Psychopharmacol 2008;28:210-213.

40. Rabinowitz J, Napryeyenko O, Burba B et al. Premorbid functioning and treatment response in recent-onset schizophrenia: prospective study with risperidone long-acting injectable. J Clin Psychopharmacol 2011;31:75-81.

41. Lieberman J. Effectiveness of antipsychotic drugs in patients with chronic schizophrenia: efficacy, safety and cost outcomes of CATIE and other trials. J Clin Psychiatry 2007;68:e04.

42. Lieberman J, Stroup T, McEvoy J et al. Effectiveness of antipsychotic drugs in patients with chronic schizophrenia. N Engl J Med 2005;353:1209-1223.

43. Ereshefsky L, Mannaert E. Pharmacokinetic profile and clinical efficacy of long-acting risperidone: potential benefits of combining an atypical antipsychotic and a new delivery system. Drugs R D 2005;6:129-137.

44. Gianfrancesco F, Rajagopalan K, Sajatovic M, Wang R. Treatment adherence among patients with schizophrenia treated with atypical and typical antipsychotics. Psychiatry Res 2006;144:177-189.

45. Becker M, Young M, Ochshorn E, Diamond R. The relationship of antipsychotic medication class and adherence with treatment outcomes and costs for Florida Medicaid beneficiaries with schizophrenia. Adm Policy Ment Health 2007;34:307-314.

46. Rummel-Kluge C, Schuster T, Peters S, Kissling W. Partial compliance with antipsychotic medication is common in patients with schizophrenia. Aust N Z J Psychiatry 2008;42:382-388.

47. Damen J, Thuresson P, Heeg B, Lothgren M. A pharmacoeconomic analysis of compliance gains on antipsychotic medications. Appl Health Econ Health Policy 2008;6:189-197.

48. Carswell C, Wheeler A, Vanderpyl J, Robinson E. Comparative effectiveness of long-acting risperidone in New Zealand: a report of resource utilization and costs in a 12-month mirror-image analysis. Clin Drug Investig 2010;30:777-787.

49. Marcus S, Olfson M. Outpatient antipsychotic treatment and inpatient costs of schizophrenia. Schizophr Bull 2008;34:173-180.

50. Sun S, Liu G, Christensen D, Fu A. Review and analysis of hospi- 
talization costs associated with antipsychotic nonadherence in the treatment of schizophrenia in the United States. Curr Med Res Opin 2007;23:2305-2312.

51. Olivares J, Rodriguez-Martinez A, Burón J, Alonso-Escolano D et al. e-STAR Study Group. Cost-effectiveness analysis of switching antipsychotic medication to long-acting injectable risperidone in patients with schizophrenia: a 12- and 24-month follow-up from the e-STAR database in Spain. Appl Health Econ Health Policy 2008;6:41-

53.
52. Lambert T, Olivares J, Peuskens J, et al. Effectiveness of injectable risperidone long-acting therapy for schizophrenia: data from the US, Spain, Australia, and Belgium. Ann Gen Psychiatry 2011;10:10.

53. Parellada E, Kouniakis F, Siurkute A, Schreiner A et al. Safety and efficacy of long-acting injectable risperidone in daily practice: an open-label, noninterventional, prospective study in schizophrenia and related disorders. Int Clin Psychopharmacol 2010;25:149-154.

Declaración de intereses:

Este estudio fue financiado por la Compañía Farmacéutica Janssen Cilag 